\title{
Parametrization of momentum and energy depositions from gravity waves generated by tropospheric hydrodynamic sources
}

\author{
N. M. Gavrilov \\ Atmospheric Physics Department, Saint Petersburg University, Petrodvorets, St. Petersburg, 198904, Russia
}

Received: 30 October 1996 / Revised: 16 April 1997 / Accepted: 22 May 1997

\begin{abstract}
The mechanism of generation of internal gravity waves (IGW) by mesoscale turbulence in the troposphere is considered. The equations that describe the generation of waves by hydrodynamic sources of momentum, heat and mass are derived. Calculations of amplitudes, wave energy fluxes, turbulent viscosities, and accelerations of the mean flow caused by IGWs generated in the troposphere are made. A comparison of different mechanisms of turbulence production in the atmosphere by IGWs shows that the nonlinear destruction of a primary IGW into a spectrum of secondary waves may provide additional dissipation of nonsaturated stable waves. The mean wind increases both the effectiveness of generation and dissipation of IGWs propagating in the direction of the wind. Competition of both effects may lead to the dominance of IGWs propagating upstream at long distances from tropospheric wave sources, and to the formation of eastward wave accelerations in summer and westward accelerations in winter near the mesopause.
\end{abstract}

\section{Introduction}

Internal gravity waves (IGWs) play an important role in the formation of the general circulation, thermal regime, and composition of the middle and upper atmosphere. According to present knowledge, the main portion of IGW energy reaches the middle and upper atmosphere from tropospheric sources. In the middle and upper atmosphere the amplitudes of waves increase, they break and produce substantial amounts of heat and momentum (Holton, 1983). Interpretation of IGW observations and inclusion of IGW effects into numerical atmospheric models requires the development of simple, but accurate numerical schemes, which give satisfactory

Correspondence to: N. M. Gavrilov description of the wave phenomena, and demand minimum computer time. Several parametrizations have been developed for the turbulent viscosity, mean flow drag and heating rates produced by dissipating IGWs (Lindzen, 1981; Matsuno, 1982; Holton, 1983; Gavrilov, 1990; Fritts and Lu, 1993; Medvedev and Klaassen, 1995; Hines, 1997). One of the main problems for the development of such parametrizations is that of the inclusion of atmospheric IGW sources.

The most developed recent theories are those of IGW generation by mountains (Pertsev, 1983; Schoeberl, 1985; Bacmeister, 1993; Nastrom and Fritts, 1992; Laprise, 1993; Lott and Teitelbaum, 1993; McLandress and McFarlane, 1993; Chunchuzov, 1994), as well as by shear instability in jet streams (Hines, 1968; Bertin et al., 1978; Fritts, 1984; Hamilton, 1984; Lindzen, 1984; Fritts and Nastrom, 1992; Sutherland et al., 1994). In the case of other tropospheric sources of IGWs, a good correlation is noticed between IGW intensity in the atmosphere and the passage of atmospheric fronts (Brodhun et al., 1974; Nagpal, 1979; Kersley and Rees, 1982; Einaudi et al., 1978; Ley and Peltier, 1978). IGWs with periods of 5-20 min and horizontal phase velocities of $10-70 \mathrm{~m} \mathrm{~s}^{-1}$ may appear at the upper edge of thunderstorms and heavy cumulus clouds (Grachev et al., 1984; Kazimirovsky, 1983; Schaning, 1992). Also among the IGW sources are convection (Townsend, 1966; Fovell et al., 1992; Manzini and Hamilton, 1993; Pfister et al., 1993a), industrial explosions (Varshavskiy and Kalikhman, 1984), disturbances due to space vehicles (Kulikov et al., 1982; Jacobson and Carlos, 1994), passage of solar terminator (Somsikov, 1995), earthquakes (Samarjiev and Pashova, 1982), volcanos (Roberts et al., 1982), sea choppiness (Golitsyn et al., 1976), cyclones and typhoons (Huang et al., 1985; Pfister et al., 1993b), and moving disturbances in the atmosphere (Grigoriev and Savina, 1983; Lipovskiy, 1981), etc.

Many of these mentioned sources are only intermittently present in the atmosphere. Besides them such important wave sources as dynamical motions of all scales should also be considered. The importance of 
these nonlinear sources lies in their permanent operation because hydrodynamic motions are an inherent feature of the atmosphere.

The generation of sound by small-scale turbulence was studied by Lighthill $(1952,1978)$. The generation of long-period inertia-gravity waves by synoptic scale motions is described by the theory of quasi-geostrophic adjustment that was developed in the dynamic meteorology beginning with Rossby, (1937), Obukhov, (1949), and Kibel, (1955). According to this theory, wave motions appear as the result of permanent competition between the violation of equilibrium of pressure and velocity and the tendency of the atmosphere to establish the quasi-geostrophic balance of these fields (Pedlosky, 1982; Gill, 1982; Gavrilov, 1987; Fritts and Luo, 1992; Medvedev and Gavrilov, 1995). The emission of mesoscale IGWs is produced by mesoscale turbulent motions (Stein, 1967; Townsend, 1965; Drobyazko and Krasilnikov, 1985; Gavrilov, 1988, 1992).

In this study the parametrization of IGW dynamic and thermal effects developed by Gavrilov (1990) involving hydrodynamic wave sources is improved. Lighthill-type mechanism of wave generation by motions of different scales is also studied. The equation of IGW energy that includes the generation of waves by mesoscale turbulence is derived. Some examples of vertical profiles of characteristics of IGW harmonics propagated to the middle atmosphere from tropospheric turbulent sources are calculated to illustrate properties of these parametrizations. Despite its simplicity the parametrization gives an accurate enough description of IGW propagation in a stratified, dissipative middle atmosphere. In the present study only the generation and propagation of monochromatic plane IGW harmonics is studied, but the parametrization developed here may serve as a basis for statistical numerical models of IGW spectrum, similar to the model developed by Medvedev and Klaassen (1995).

\section{Mathematical model}

\subsection{Wave generation by turbulence}

Turbulent motions of different scales permanently occur in the atmosphere. Fluctuations with time scales up to several minutes are attributed to microscale turbulence, time scales from minutes to several hours belong to mesoscale turbulence, and a stochastic regime of synoptic scale irregular motions may be regarded as macro-scale turbulence (Monin and Yaglom, 1965). Microscale turbulent motions are almost isotropic and do not respond to the gravity and Coriolis forces acting in the Earth's atmosphere. Gravity is essential for the mesoscale turbulence, and eddies with time scales longer than several hours are influenced by the Coriolis force, which is very important for macroscale turbulent vortexes.

On the other hand, the atmosphere has a broad spectrum of wave motions from high frequency acoustic waves up to planetary waves with periods of several days. Because atmospheric hydrodynamic equations are nonlinear, turbulent and wave motions are not independent: an energy exchange exists that leads, in particular, to the generation of waves by turbulence. To describe the latter process let us consider a conventional set of inviscid adiabatic hydrodynamic equations for a plane rotating atmosphere (see Gill, 1982):

$$
\begin{aligned}
\frac{\partial \rho}{\partial t}+\nabla(\rho \mathbf{v}) & =0 ; \\
\rho \frac{\mathrm{d} \mathbf{v}}{\mathrm{d} t}+2 \rho f \mathbf{e}_{z} \times \mathbf{v}+\nabla p+\rho g \mathbf{e}_{z} & =0 ; \\
\frac{\mathrm{d} p}{\mathrm{~d} t}-c_{s}^{2} \frac{\mathrm{d} \rho}{\mathrm{d} t} & =0,
\end{aligned}
$$

where $p, \rho, \mathbf{v}$ are pressure, density, and velocity; $c_{s}$ is the speed of sound; $g$ is the acceleration due to gravity; $f$ is the Coriolis parameter; $\mathbf{e}_{z}$ is a unit vertical vector; $\mathrm{d} / \mathrm{d} t=\partial / \partial t+\mathbf{v} \cdot \nabla$.

Let us consider a motionless atmosphere with background values of $p_{0}, \rho_{0}$ varying in altitude $z$ according to the barometric law, and $\mathbf{v}_{0}=0$. Dynamic fluctuations $\mathbf{v}_{d}, \rho_{d}=\rho-\rho_{0}, p_{d}=p-p_{0}$ are usually small, so that $\left|\rho_{d} / \rho_{0}\right| \ll 1,\left|p_{d} / p_{0}\right| \ll 1$ and $\left|\mathbf{v}_{d}\right| / c_{s 0} \ll 1$. Let Mach's number $M$ be a measure of the largest value of $\left|\mathbf{v}_{d}\right| / c_{s 0}$. When $M$ is small, one should search for the solution to the Eq. (1) as series expansions with respect to $M$ :

$$
\begin{aligned}
& \rho=p_{0}+M p_{t}+M^{2} p^{\prime}+\cdots ; \\
& \rho=\rho_{0}+M \rho_{t}+M^{2} \rho^{\prime}+\cdots ; \\
& \mathbf{v}=M \mathbf{v}_{t}+M^{2} \mathbf{v}^{\prime} ; c_{s}^{2}=c_{s 0}^{2}+M c_{s t}^{2}+M^{2} c_{s}^{\prime 2}+\cdots
\end{aligned}
$$

Substituting Eq. (2) into (1) and equating coefficients at the same powers of $M$ to zero, the following set of equations may be produced for the components of hydrodynamic fields of the order of $M^{2}$ :

$$
\frac{\mathrm{d}_{0} \rho^{\prime}}{\mathrm{d} t}+\nabla\left(\rho_{0} \mathbf{v}^{\prime}\right)=\rho_{0} q
$$

$$
\rho_{0} \frac{\mathrm{d}_{0} \mathbf{v}^{\prime}}{\mathrm{d} t}+2 \rho_{0} f \mathbf{e}_{z} \times \mathbf{v}^{\prime}+\nabla p^{\prime}+\rho^{\prime} g \mathbf{e}_{z}=\rho_{0} \mathbf{r}
$$

$\frac{\mathrm{d}_{0} p^{\prime}}{\mathrm{d} t}+\mathbf{v}^{\prime} \cdot \nabla p_{0}-c_{s 0}^{2}\left(\frac{\mathrm{d}_{0} \rho^{\prime}}{\mathrm{d} t}+\mathbf{v}^{\prime} \cdot \nabla \rho_{0}\right)=\rho_{0} h$.

Right hand parts of the Eq. (3) include the hydrodynamic sources of mass $q$, momentum $\mathbf{r}$ and energy $h$ that describe the generation of the second order motions by the motions of the first order with respect to $M$ :

$$
\begin{aligned}
& q=-\rho_{0}^{-1} \nabla\left(\rho_{t} \mathbf{v}_{t}\right) \\
& \mathbf{r}=-\rho_{0}^{-1}\left(\rho_{t} \frac{\mathrm{d}_{0} \mathbf{v}_{t}}{\mathrm{~d} t}+\rho_{0} \mathbf{v}_{t} \cdot \nabla \mathbf{v}_{t}\right) \\
& h=-\rho_{0}^{-1}\left(\mathbf{v}_{t} \cdot \nabla p_{t}-c_{s 0}^{2} \mathbf{v}_{t} \cdot \nabla \rho_{t}\right)+c_{s t}^{2}\left(\frac{\mathrm{d}_{0} \rho_{t}}{\mathrm{~d} t}+\mathbf{v}_{t} \cdot \nabla \rho_{0}\right) .
\end{aligned}
$$

At the time scales corresponding to acoustic-gravity waves, the main contribution into the first order components is made by irregular turbulent motions, 
which may be treated as three-dimensional and solenoidal at small scales. They become two-dimensional and quasi-geostrophic at medium and large scales (Drobyazko and Krasilnikov, 1985; Gavrilov, 1988; Medvedev and Gavrilov, 1995).

Differentiating the first Eq. of (3) with respect to $t$ and using the relation $p^{\prime}=c_{s 0}^{2} \rho^{\prime}$ for adiabatic acoustic waves, we have the following wave equation for the case of $\mathbf{v}_{0}=0$ and solenoidal $\mathbf{v}_{t}$ :

$\frac{\partial^{2}\left(\rho^{\prime} / \rho_{0}\right)}{\partial t^{2}}-c_{s 0}^{2} \Delta \frac{\rho^{\prime}}{\rho_{0}}=\frac{\partial^{2} v_{t i} v_{t j}}{\partial x_{i} \partial x_{j}}$.

This is exactly the equation obtained by Lighthill (1952) that describes the generation of sound by microscale turbulence. For the case of IGW generation by mesoscale turbulence one may also obtain from Eq. (3) inhomogeneous wave equations with wave sources in their right hand parts. Those equations are complicated and are derived by Stein (1967), Drobyazko and Krasilnikov (1985), Gavrilov (1987).

\subsection{Wave energy equation}

To study IGW generation by hydrodynamic sources we use here another approach based on the consideration of the equation of wave energy. It is known that the specific density of wave energy $E_{w}$ consists of two parts: kinetic, $E_{w k}$, and potential, $E_{w p}$, which are given by the following expressions (see Dikiy, 1969):

$$
E_{w}=E_{w k}+E_{w p} ; E_{w k}=\rho_{0}\left\langle\left|\mathbf{v}^{\prime}\right|^{2}\right\rangle / 2
$$

$$
E_{w p}=\left\langle p^{\prime 2}\right\rangle / 2 \gamma p_{0}+\left\langle\left(p^{\prime}-c_{s 0}^{2} \rho^{\prime}\right)^{2}\right\rangle / 2 \gamma b p_{0},
$$

where the sign \langle\rangle denotes averaging over wave period; $\gamma=c_{p} / c_{v} ; b=(\gamma-1)+g^{-1} \partial c_{s 0}^{2} / \partial z ; c_{p}$ and $c_{v}$ are the specific heat capacities at constant pressure and volume, respectively. Multiplying Eq. (3) by $\rho^{\prime}, \mathbf{v}^{\prime}$ and $p^{\prime}$ respectively, one may derive the equation for $E_{w}$, in the form of:

$$
\begin{aligned}
& \frac{\mathrm{d}_{0} E_{w}}{\mathrm{~d} t}=-\frac{\partial F_{w \alpha}}{\partial x_{\alpha}}-\rho_{0} v_{\beta 0} a_{w \beta}+\rho_{0} Q ; \\
& a_{w \beta}=-\frac{1}{\rho_{0}} \frac{\partial \rho_{0}\left\langle v_{\alpha}^{\prime} v_{\beta}^{\prime}\right\rangle}{\partial x_{\alpha}},
\end{aligned}
$$

where $a_{w \beta}$ is the acceleration of the mean flow by waves, $F_{w \alpha}$ is the total wave energy flux, which includes transport by the mean wind (see Gavrilov, 1990):

$F_{w \alpha}=\left\langle p^{\prime} v_{\alpha}^{\prime}\right\rangle+\rho_{0} v_{\beta 0}\left\langle v_{\alpha}^{\prime} v_{\beta}^{\prime}\right\rangle$

where repeated indices assume summation. The quantity $Q$ in Eq. (7) is the rate of wave energy production by hydrodynamic sources of momentum, mass and heat per unit atmospheric volume:

$Q=\rho_{0}^{-1}\left\langle\rho_{0} \mathbf{r} \cdot \mathbf{v}^{\prime}+q p^{\prime}+h\left[\frac{p^{\prime}(b+1)}{c_{s 0}^{2} b}-\frac{\rho^{\prime}}{b}\right]\right\rangle$

In this study we will consider a stationary and horizontally inhomogeneous model, when only the vertical component of the total energy flux, $F_{w z}$, gives a contribution to Eq. (7). It can be shown that in such models $F_{w z}=$ const in the absence of irreversible and dissipative processes (see Gavrilov, 1990), consequently, variations of the mean wind and temperature cannot change the value of $F_{w z}$. Also, we will find later that $F_{w z}$ is proportional to the vertical component of the wave flux of horizontal momentum, $F_{m z}$.

\subsection{The strength of hydrodynamic IGW sources}

The formula (9) for the rate of the wave energy production $Q$ may be simplified using IGW polarization relations, which are most simple for the short lowfrequency IGW harmonics with $m^{2} \gg 1 / 4 H^{2}$ and $\omega^{2} \ll N^{2}$, where $m$ and $\omega$ are the vertical wave number and intrinsic frequency, respectively; $N$ is the BruntVaisala frequency; $H$ is the atmospheric scale height. If the axis $x$ is directed parallel to the horizontal wave vector of a plane monochromatic IGW harmonic, for the concerned IGWs (see Gavrilov et al., 1996) the following dispersion equation may be obtained:

$m^{2}=N^{2} k^{2} /\left(\omega^{2}-f^{2}\right)$,

together with the polarization relations:

$\frac{v^{\prime}}{u^{\prime}} \simeq i \frac{f}{\omega} ; \frac{w^{\prime}}{u^{\prime}} \simeq-\frac{\omega \delta}{N} ; \frac{p^{\prime}}{p_{0} u^{\prime}} \simeq \frac{\gamma \omega \delta^{2}}{k c_{s 0}^{2}} ; \frac{\rho^{\prime}}{\rho_{0} u^{\prime}} \simeq i \frac{N \delta}{g} ;$

where $k$ is the horizontal wave number; $\delta=\left(1-f^{2} / \omega^{2}\right)^{1 / 2} ; u, v, w$ are the velocity components along horizontal axes $x, y$ and vertical axis $z$, respectively. For stationary and horizontally homogeneous model we can make Fourier transformation of all of IGW fields and wave sources. The expression (9) shows that for each monochromatic IGW harmonic depending on $t$ and $x$ as

$u^{\prime}=U e^{i(\sigma t-i k x)}$

the wave source $Q$ does not equal zero only when spectra of the sources $\mathbf{r}, q$ and $h$ also contain harmonics with an observable frequency $\sigma$ and horizontal wave number $k$ :

$r_{\alpha}=r_{\alpha m} e^{i\left(\sigma t-k x+\varphi_{\alpha}\right)} ; \quad \alpha=x, y, z ;$
$q=q_{m} e^{i\left(\sigma t-k x+\varphi_{q}\right)} ; \quad h=h_{m} e^{i\left(\sigma t-k x+\varphi_{h}\right)}$,

where $r_{\alpha m}, q_{m}, h_{m}$ are amplitudes, and $\varphi_{\alpha}, \varphi_{q}, \varphi_{h}$ are the phase shifts between variations of respective wave source and $u^{\prime}$. Putting the real parts of Eqs. (11)-(13) into Eq. (9), we have

$$
\begin{aligned}
Q=s U & ; s=1 / 2\left[r_{x m} \cos \varphi_{x}+r_{y m}\left(f \sin \varphi_{y}\right) / \omega\right. \\
& \left.-r_{z m} \frac{\omega \delta}{N} \cos \varphi_{z}-h_{m} \frac{N \delta}{b g} \sin \varphi_{h}+q_{m} \frac{\gamma \omega \delta^{2}}{k c_{s 0}^{2}} \cos \varphi_{q}\right] .
\end{aligned}
$$

It can be seen that the rate of wave energy production is governed by the value of parameter $s$, which has the dimension of force acting upon a unit mass of atmo- 
spheric gas. According to evaluations presented by Drobyazko and Krasilnikov (1985) and Gavrilov (1987), the main contribution to the IGW generation comes from the first term in square brackets of Eq. (14), which is connected with quasi-two-dimensional mesoscale motions, so $s \sim \bar{r}_{q} \sim u_{q} \partial u_{q} / \partial q$, where $u_{q}$ and $\partial u_{q} / \partial q$ are the scales of horizontal velocity and its horizontal gradient, respectively, $q=x, y$. In accordance with the tables of characteristics for the tropospheric meteorological values (Belov, 1975) we assume that $u_{q} \sim 10^{1} \mathrm{~m} \mathrm{~s}^{-1}, \partial u_{q} / \partial q \sim 10^{-5}-10^{-4} \mathrm{~s}^{-1}$, and therefore $\bar{r}_{q} \sim 10^{-5}-10^{-3} \mathrm{~m} \mathrm{~s}^{-2}$.

The order of magnitude of $s \sim \bar{r}_{q}$ may be estimated from the data about tropospheric mesoscale turbulence. Lilly (1983) found that observed energy spectra of tropospheric mesoscale turbulence, which is supposed to be two-dimensional, may be approximated by the formula

$E_{t}(k)=C\left(\mathrm{~d} E_{t} / \mathrm{d} t\right)^{2 / 3} k^{-5 / 3}$

where $k$ is the horizontal wave number, $\mathrm{d} E_{t} / \mathrm{d} t$ is the rate of energy injection into turbulence, and $C$ is a dimensionless constant. Assuming that the spectrum of $\bar{r}_{q}$ is proportional to $k E_{t}(k)$, we may anticipate that the spectra of turbulent wave sources could be proportional to $k^{-2 / 3}$ in the spectral range $k \sim 10^{-6}-10^{-3} \mathrm{~m}^{-1}$, which corresponds to the horizontal scales of 3-3000 km (Lilly, 1983). Multiplying Eq. (15) by $k$ and integrating over the mentioned range of $k$, we may get the estimation of the integral intensity of $\bar{r}_{q} \sim 9 \cdot 10^{-4} \mathrm{~m} \mathrm{~s}^{-2}$ using the values of $C \sim 7$ and $\mathrm{d} E_{t} / \mathrm{d} t \sim 10^{-5} \mathrm{~m}^{2} \mathrm{~s}^{-3}$ proposed by Lilly (1983)

Gage (1979) has also found evidence for $k^{-5 / 3}$ law validity in the inertial subrange of two-dimensional turbulence. He gave experimental data for the variability, $D(\tau)$ of wind with time at a fixed point and altitude:

$D(\tau)={\overline{\left\{[v(t)-v(t+\tau)]^{2}\right.}}^{1 / 2}$,

where $\tau$ is the lag time. One may consider Eq. (16) as a frequency filter estimating the doubled amplitude of Fourier harmonic with a period $T \sim 2 \tau$. Assuming $\bar{r}_{q} \sim k D^{2}(T / 2) / 2$ and using the relation $k=\sigma / v_{0}$ for frozen turbulence (see Gage, 1979), the estimation $\bar{r}_{q}(\sigma) \sim \sigma D^{2}(\pi / \sigma) / 2 v_{0}$ can be made. High-resolution balloon measurements (Gage, 1979) give $D^{2} \sim 9-2 \mathrm{~m}^{2} \mathrm{~s}^{-2}$ at $\sigma \sim 6 \cdot 10^{-4}-6 \cdot 10^{-3} \mathrm{~s}^{-1}$ for zonal wind component at the altitudes $0-20 \mathrm{~km}$. With $v_{0} \sim 10 \mathrm{~m} \mathrm{~s}^{-1}$ these values of $D^{2}$ lead to $\bar{r}_{q} \sim$ $(3-6) \cdot 10^{-4} \mathrm{~m} \mathrm{~s}^{-2}$ at $\sigma \sim 6 \cdot 10^{-4}-6 \cdot 10^{-3} \mathrm{~s}^{-1}$.

Radar measurements of the variability of the meridional wind at altitudes of $5-13 \mathrm{~km}$ (Gage, 1979) give values of $D^{2} \sim 15-100 \mathrm{~m}^{2} \mathrm{~s}^{-2}$ at $\sigma \sim 10^{-2}-3 \cdot 10^{-4} \mathrm{~s}^{-1}$, also $v_{0} \sim 30 \mathrm{~m} \mathrm{~s}^{-1}$. They lead to $\bar{r}_{q} \sim 5 \cdot 10^{-4}-2$. $10^{-3} \mathrm{~m} \mathrm{~s}^{-2}$ for $\sigma \sim 3 \cdot 10^{-4}-10^{-2} \mathrm{~s}^{-1}$. All these estimations of $\bar{r}_{q}$ for tropospheric mesoscale turbulence give consistent orders of magnitude of $s \sim \bar{r}_{q} \sim 10^{-4}$ $-10^{-3} \mathrm{~m} \mathrm{~s}^{-2}$ in the troposphere.

\subsection{IGW energetic characteristics}

Using Eqs. (10) and (11) for IGWs with $\omega^{2} \gg f^{2}$ and $m^{2} \gg 1 / 4 H^{2}$, the vertical component of total energy flux $F_{w z}$ in Eq. (7) may be expressed as follows (see Gavrilov, 1990):

$F_{w z}=-\rho_{0} \sigma U^{2} /(2 m)$,

where $m<0$ corresponds to downward propagation of IGW phase and upward propagation of wave energy. Equation (7) does not include IGW dissipation. Taking these processes into account and using the approach of Gavrilov (1990) with the account of Eqs. (14) and (17), one may obtain instead of Eq. (7) the wave energy equation for the stationary and horizontally homogeneous model that has the following form:

$-\frac{1}{2} \frac{\partial}{\partial z}\left(\frac{\sigma}{m} \rho_{0} U^{2}\right)=\rho_{0} \frac{\sigma}{\omega}\left(s U-\frac{N_{d}}{2} U^{2}\right)$,

where $N_{d}$ is the rate of IGW dissipation due to turbulent and molecular viscosity and heat conduction, radiative heat exchange, and ion drag (see Gavrilov, 1990). Introducing a new variable $S=2\left|F_{w z}\right|^{1 / 2}$, Eq. (18) may be transformed into the following form:

$\frac{\partial S}{\partial z}+\frac{N_{d} m}{2 \omega} S=\frac{s\left(\sigma m \rho_{0}\right)^{1 / 2}}{\omega} \cdot \operatorname{sign}\left(F_{w z}\right)$.

This is an inhomogeneous linear equation describing the IGW generation by the hydrodynamic sources of momentum, mass and heat. After solving Eq. (19), the vertical total flux of wave energy and wave amplitude may be calculated as follows:

$F_{w z}=-\frac{S^{2}}{2} \operatorname{sign}(m) ; \quad U=\left|\frac{m}{\sigma \rho_{0}}\right|^{1 / 2} S$.

Some characteristics are essential for studies and parametrizations of IGW effects and their incorporation into numerical models of atmospheric general circulation. Among those IGW characteristics are the vertical wave flux of horizontal momentum, $F_{m z}$, wave acceleration of the mean flow, $a_{w}=a_{w x}$, and the wave heat flux divergence, $\varepsilon_{w}$. Using relations (10), (11) and (17), we have for IGWs with $N^{2} \gg \omega^{2} \gg f^{2}$ and $m^{2} \gg 1 / 4 H^{2}$ that:

$F_{m z}=\rho_{0}\left\langle u^{\prime} w^{\prime}\right\rangle=F_{w z} / c$,

where $c=\sigma / k$ is the observable IGW horizontal phase speed. Remembering the discussion of $F_{w z}$ in Sect. 2.2, it can be seen from Eq. (21) that in the absence of irreversible and dissipative processes the condition $F_{w z}=$ const (see Gavrilov, 1990) leads to the condition $F_{m z}=$ const for an IGW harmonic with given $\sigma$ and $k$. Expressions for $a_{w}$ and $\varepsilon_{w}$ were obtained by Gavrilov (1990) and have the following form:

$a_{w}=\frac{N_{d}}{c-u_{0}} \frac{U^{2}}{2} ; \varepsilon_{w}=\frac{c}{c-u_{0}} N_{d} \frac{U^{2}}{2}$.

The Eqs. (18)-(22) allow us to calculate vertical profiles of IGW characteristics for arbitrary vertical 
distributions of atmospheric background temperature, wind, rates of dissipation, and strength of wave hydrodynamic sources.

\section{$2.5 I G W$ dissipation}

Equation (19) contains the rate of IGW dissipation, $N_{d}$, due to turbulent and molecular viscosity and heat conduction, ion drag, and radiative heat exchange (see Gavrilov, 1990). One of the main mechanisms of IGW dissipation in the atmosphere is turbulence. It may be generated as a result of wave breaking caused by dynamical and convective instability (Lindzen, 1981) or nonlinear destruction of a primary IGW into secondary wave harmonics (Weinstock, 1982). Also, IGW may dissipate due to background atmospheric turbulence produced by other sources. In this study the coefficient of turbulent viscosity is represented by the following sum:

$K=K_{0}+K_{i}+K_{n}$,

where $K_{0}, K_{i}$, and $K_{n}$ are the background value, and contributions of IGW instability and nonlinear wave interactions, respectively. The value of $K_{i}$ is zero up to the altitude at which IGW becomes unstable, and $K_{i}$ is calculated using the semi-empirical model of Gavrilov and Yudin (1992) above this altitude.

Several studies of nonlinear destruction of the primary IGW into secondary wave harmonics were made for resonant and nonresonant wave-wave interactions (Weinstock, 1982, 1990; Dong and Yeh, 1988; Rozenfeld, 1983; Sonmor and Klaassen, 1996). Some papers contain expressions for the effective coefficients of turbulent viscosity produced by a spectrum of secondary wave harmonics generated by a primary IGW. Weinstock (1982) obtained a formula for the nonlinear decay rate, $d_{R}$, of the amplitude of monochromatic IGW harmonic (see also Medvedev and Klaassen, 1995). The corresponding coefficient of turbulent viscosity $K_{n W}=2 d_{R} / m^{2}$ has the following form:

$K_{n W}=\frac{(2 \pi)^{1 / 2} N \omega^{2}}{m^{3} V} \exp \left(-\frac{N^{2}}{2 m^{2} V^{2}}\right)$,

where $V^{2}$ is the integral variance of horizontal velocity produced by secondary wave harmonics. Weinstock (1990) and Medvedev and Klaassen (1995) supposed that only harmonics with $\omega^{\prime}>\omega$ and $m^{\prime}>m$ may contribute to $V^{2}$. Assuming slopes $\omega^{-5 / 3}$ and $m^{-3}$ for the respective spectra of secondary waves, we can estimate that $V^{2} \sim 0.8 U^{2}$. Another formula for the effective coefficient of turbulent viscosity produced by the nonlinear secondary waves was obtained by Rozenfeld (1983), who used expansion of wave fields with respect to a small parameter related to the wave amplitude. Using Eqs. (10) and (11), we may write his formula in the following form:

$K_{n R}=2.28 \frac{\omega}{m^{3} H} \frac{U^{2}}{c_{s 0}^{2}}$, where $H$ and $c_{s 0}$ are the scale heights and the sound speed, respectively.

Equation (19) is written for a single IGW harmonic. In the case of IGW spectrum, Eq. (19) may be solved for each harmonic, and then values (20)-(23) should be integrated over considered ranges of $\sigma$ and $k$.

\section{Results of calculation}

\subsection{Analytical solution}

The essential features of the vertical distributions of the strength of tropospheric wave sources (already discussed) may be approximated by the following formula:

$s(z)=s_{m} \exp \left[-\left(z-z_{m}\right)^{2} / d^{2}\right]$,

where $z_{m}$ and $d$ are, respectively, the height of maximum and the half width of the layer in which the generation of IGWs takes place. The analytical solution to Eqs. (19), (26) may be obtained for the simplest model of isothermal atmosphere with constant values of the mean wind and IGW dissipation rate. When $z_{m} \gg d$ and $z-z_{m} \gg d$ the solution has the following form:

$$
\begin{gathered}
S(z)=\frac{d s_{m}\left|\sigma m \rho_{0 m}\right|^{1 / 2}}{\omega} \exp \left[\frac{1}{16}\left(\frac{d}{H}-\frac{d}{L_{d}}\right)^{2}-\frac{z-z_{m}}{2 L_{d}}\right], \\
L_{d}=\frac{\omega}{N_{d} m},
\end{gathered}
$$

where $\rho_{0 m}$ is the background density at the level $z_{m}$. Formulae (20) and (27) show that $U$ is proportional to $s_{m}$, and $F_{w z}$ is proportional to $s_{m}^{2}$. For characteristic values of $z_{m}=10 \mathrm{~km}, d=2 \mathrm{~km}, H=7 \mathrm{~km}$ in tropospheric hydrodynamic wave sources, and for the IGW harmonic with $\omega=10^{-3} \mathrm{~s}^{-1}$ the values of $U \sim 10-50 \mathrm{~m} \mathrm{~s}^{-1}$ observed at the altitudes near $100 \mathrm{~km}$ may be obtained if one specifies in Eq. (19) $s_{m} \sim(8-42) \times 10^{-6} \mathrm{~m} \mathrm{~s}^{-2}$ at $m=10^{-3} \mathrm{~m}^{-1}, \quad$ and $s_{m} \sim(8-40) \times 10^{-5} \mathrm{~m} \mathrm{~s}^{-2}$ at $m=10^{-4} \mathrm{~m}^{-1}$.

These values of $s_{m}$ have smaller orders of magnitude than the estimations of $\bar{r}_{q}$ for tropospheric mesoscale turbulence made in Sect. 2.3. Thus mesoscale irregular motions existing in the troposphere are strong enough to provide IGW amplitudes of the same order of magnitude as the amplitudes observed in the middle and upper atmosphere, even if only a fraction of wave energy generated in the troposphere is capable of reaching high altitudes. Permanent IGW generation by mesoscale turbulence may provide a background level of wave intensity in the atmosphere. Other wave sources (see introduction) may make additional contribution, which could be larger or smaller depending on the strength of respective wave sources.

\subsection{Numerical solution}

Formula (27) is valid only for altitudinally constant values of temperature, mean wind, and $N_{d}$. In a realistic 
atmosphere those values as well as $s(z)$ have a complicated dependence upon altitude, which requires a numerical solution to Eq. (19). For our calculations we use the vertical profiles of the mean temperature, wind, and the rates of IGW dissipation due to radiative heat exchange and ion drag according to Gavrilov and Yudin, (1987). The coefficient of turbulent viscosity is represented by Eq. (23), where we use a typical background value in stratosphere of $K_{0} \sim 10^{0} \mathrm{~m}^{2} \mathrm{~s}^{-1}$. The value of $K_{i}$ in Eq. (23) is equal to zero up to the level of IGW breaking, and it is calculated using the semiempirical model by Gavrilov and Yudin (1992) above the breaking altitude. The term $K_{n}$ in Eq. (23) represents a contribution from the alternative mechanism of turbulence in the atmosphere, which is caused by nonlinear destruction of the primary IGW into secondary wave harmonics (Weinstock, 1982). For $K_{n}$ in Eq. (23) we use the formulae (24) and (25).

The formula for $N_{d}$ in Eq. (19) contains the coefficient of turbulent heat conduction $K_{t}=K / \mathrm{Pr}$, where $\mathrm{Pr}$ is the Prandtl number. In the case of IGW convective instability the formation of turbulent layers, may be expected and $\operatorname{Pr}>1$ (Gavrilov and Yudin, 1992). One may also expect the background turbulence and the system of nonlinear secondary wave harmonics to be more homogeneous with $\operatorname{Pr} \sim 1$. In present calculations we use the value $\operatorname{Pr}=1$ for all kinds of turbulence. It might slightly overestimate IGW dissipation, but cannot change qualitative results of our calculations.

Figure 1 shows the vertical profiles of amplitudes and values of $K$ produced by IGW harmonic propagated from tropospheric hydrodynamic source in an isothermal windless atmosphere for different mechanisms producing turbulence in the atmosphere by IGWs. The dotted line in Fig. 1 shows that, without dissipation, the amplitude grows exponentially with altitude. The nonlinear destruction of the IGW into secondary harmonics leads to wave energy dissipation. Dashed and thin solid lines in Fig. 1 correspond to the formulae (24) and (25), respectively. It may be seen that formula (24) gives small
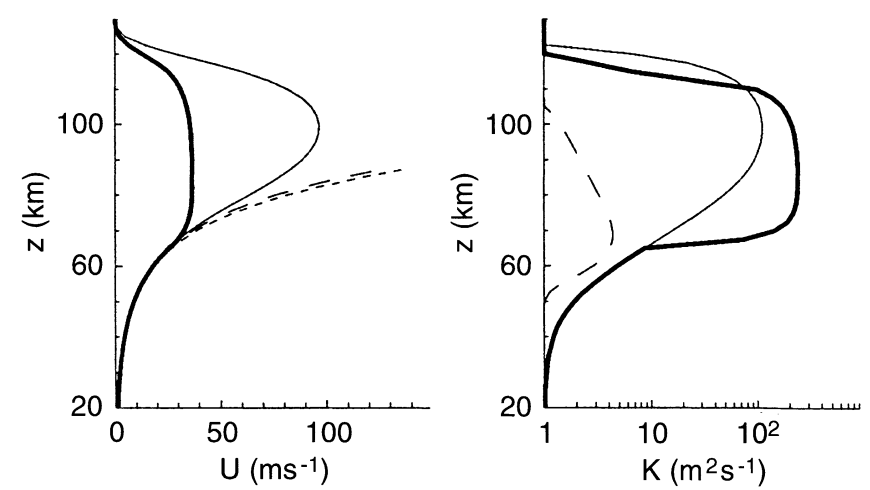

Fig. 1. Vertical profiles of the amplitude (left) and turbulent viscosity (right) calculated for the IGW harmonic with $\sigma=10^{-3} \mathrm{~s}^{-1}$ and $c=30 \mathrm{~m} \mathrm{~s}^{-1}$ propagated from tropospheric hydrodynamic source with $s_{m}=10^{-4} \mathrm{~m} \mathrm{~s}^{-2}, z_{m}=10 \mathrm{~km}$, and $d=2 \mathrm{~km}$ in isothermal windless atmosphere with $T_{0}=230 \mathrm{~K}$ for the values of $K=0$ (dotted lines), $K=K_{0}+K_{n R}$ (thin solid lines), $K=K_{0}+K_{n W}$ (dashed lines), and $K$ described by Eq. (23) in solid (thick lines) values of $K_{n}$, and the rate at which wave amplitude grows is practically the same as without dissipation (see dashed lines in Fig. 1). Thin solid lines in Fig. 1 for formula (25) have maxima near the altitude $100 \mathrm{~km}$. The parameter of IGW supersaturation $a=U /\left|c-u_{0}\right|$ is about $a \sim 3$ here. This value seems to be nonrealistic, because at $a \sim 1$ the wave becomes convectively unstable, breaking and saturated (see thick lines in Fig. 1 and Gavrilov and Yudin, 1992).

Lindzen and Forbes (1983) presumed that hypothetical IGW instability existed, which may provide smooth growth of turbulent viscosity from zero at low altitudes to the large values corresponding to IGW saturation above the wave breaking level. One of the mechanisms possible of such additional turbulence may be the nonlinear destruction of primary IGW into the spectrum of irregular secondary harmonics. Figure 1 shows that formula (25) gives a smoother transition of $K$ from $K=K_{0}$ at $z \sim 20 \mathrm{~km}$ to $K \sim K_{i}$ above the breaking level. So we use Eq. (25) in the present model.

The presence of the mean wind makes differences for IGWs propagating upstream and downstream. Figure 2 depicts the characteristics of IGW harmonics propagating in the isothermal atmosphere with constant values of $u_{0}$ from the tropospheric sources with the same values of $s$ for both directions. One may see that at low altitude amplitude, total wave energy flux, and other parameters in Fig. 2 are larger for the IGW propagating in the direction of mean flow. At high altitudes the IGW going
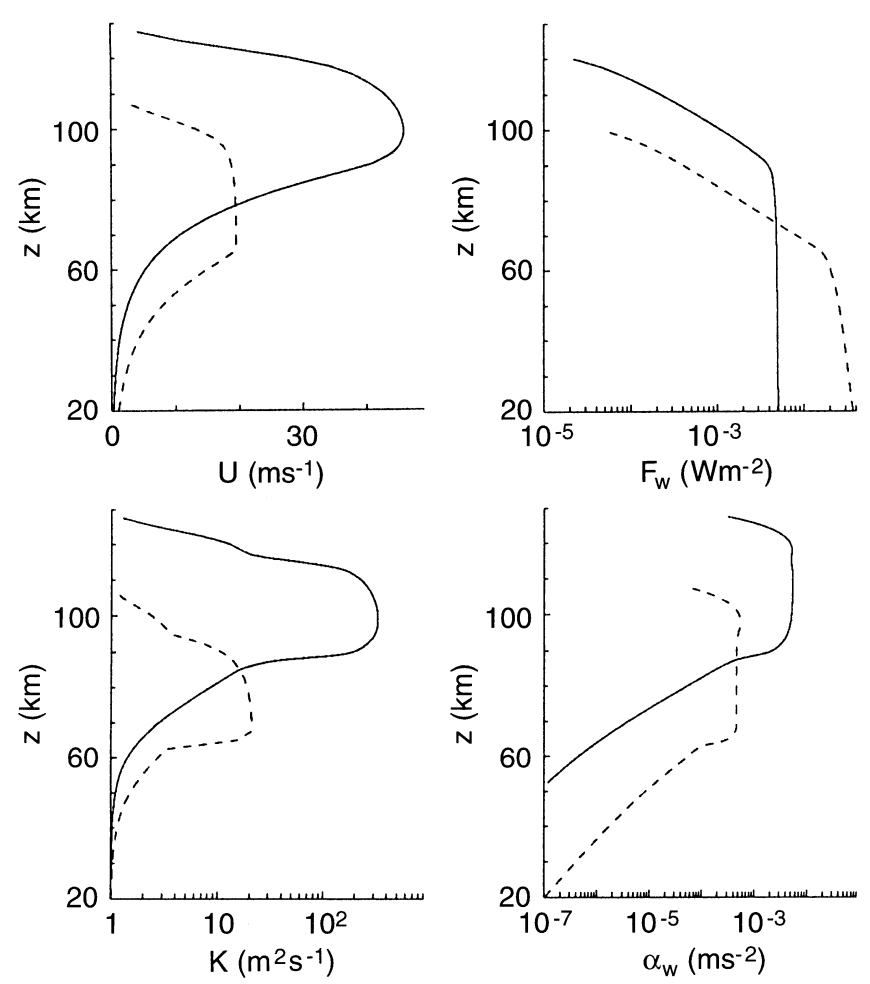

Fig. 2. Vertical profiles of IGW amplitude, $U$, total vertical energy flux, $F_{w}$, turbulent viscosity, $K$, and wave drag, $a_{w}$, calculated for the same model as Fig. 1, but including constant mean wind $u_{0}=10 \mathrm{~m} \mathrm{~s}^{-1}$ for the waves propagated in the direction of the mean flow (dashed lines), and opposite to it (solid lines) 
in the opposite direction to the mean flow becomes more intensive. To understand such behaviour the dual action of the mean wind on the IGWs should be considered: i.e. the wave-mean flow interaction, and the change of the vertical wave number.

When IGW propagates in shear flow, the interaction of waves with the mean flow may increase or decrease the wave intensity (see Gavrilov et al., 1995). According to Eq. (7), the specific rate of energy influx from mean flow to waves is equal to $u_{0} \partial\left(\rho_{0} \overline{u^{\prime} w^{\prime}}\right) / \partial z$ (see also Gavrilov and Shved, 1975). In the region of the active tropospheric wave source near $z \sim z_{m} \sim 10 \mathrm{~km}$ the modulus of $\left|\rho_{0} \overline{u^{\prime} w^{\prime}}\right|$ increases. This means that the interaction with the mean flow makes an increase in the intensity of IGWs propagating in the direction of the mean flow, and the decrease in the intensity of upstream IGWs. Thus, at the same strength of $s$ the amplitude of the downstream wave is larger than the amplitude of the upstream IGW. This difference is expressed mathematically by the appearance of the factor $\sigma / \omega$ in the right hand part of Eq. (18) and explains the dominance of downstream IGWs at low altitudes near the wave source in Fig. 2.

On the other hand, above the region of the wave source the influence of $u_{0}$ leads to an increase in $\omega$ for the upstream IGW, and to a decrease in $\omega$ for the downstream wave. According to the IGW dispersion equation the downstream IGW has a larger value of $m$, which corresponds to larger dissipation of wave energy. Also, the downstream IGW breaks and becomes saturated at smaller altitudes than the upstream wave. The larger dissipation of the downstream IGW may explain the dominance of the upstream wave in the upper part of Fig. 2. In the region of strong dissipation the wave acceleration of the mean flow has the same direction as the wave horizontal phase speed. So, the dominance of the upstream wave in Fig. 2 means the dominance of the drag of the mean flow by IGWs.

To take account of the influence of realistic mean temperature and wind we made calculations using profiles of those values for January and July that are shown in Fig. 3. The results of the computations are presented in Figs. 4-6 for IGW harmonics with different
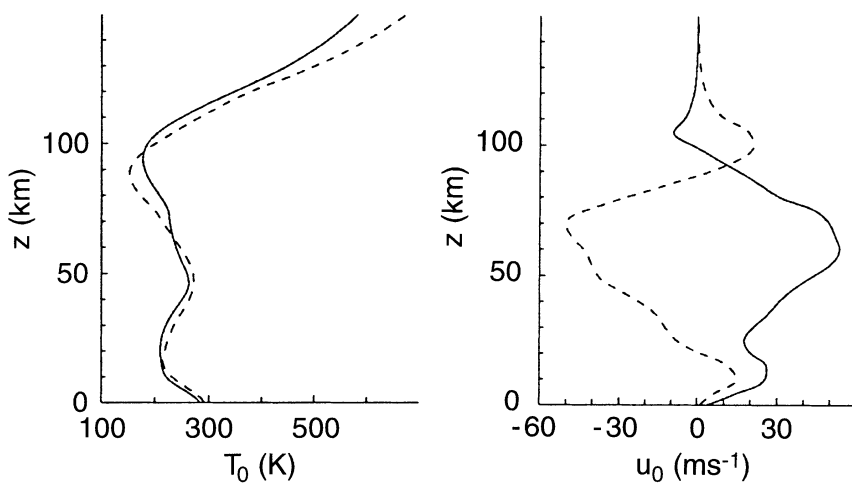

Fig. 3. Vertical profiles of the mean temperature, $T_{0}$, and zonal wind, $u_{0}$, for January (solid lines) and July (dashed lines) at latitude $45^{\circ} \mathrm{N}$ (Hedin, 1991). Positive values of $u_{0}$ correspond to the eastern direction horizontal phase speeds, and propagating to the east and west. In all cases we presume the strength of tropospheric IGW source, $s$, to be the same for opposite directions. Figure 4 presents the vertical variations of IGW parameters for the harmonic where $c= \pm 30 \mathrm{~m} \mathrm{~s}^{-1}$ that is much smaller then the peak values of the mean wind in the stratosphere in both seasons (see Fig. 3). Strong dissipation of the IGW harmonic propagating in the direction of the mean wind in the stratosphere (eastward in winter and westward in summer) caused by their smaller vertical wavelengths and breaking near the critical levels (see earlier) makes all their parameters so small that they are not visible in Fig. 4. Hence at the same $s$ the IGW harmonics propagating opposite to the mean wind become larger in the middle and upper atmosphere, and produce the wave accelerations directed to the west in winter, and to the east in summer near the altitude $100 \mathrm{~km}$ in Fig. 4.

Figure 5 shows the same profiles as Fig. 4, but for the IGW harmonics with $c= \pm 60 \mathrm{~m} \mathrm{~s}^{-1}$ that is slightly larger than the largest $u_{0}$ in Fig. 3. Considerable dissipation of the eastward harmonic in the stratosphere leads to the dominance of the westward IGW and wave accelerations in winter (see Fig. 5) provided the tropospheric wave sources are isotropic. In summer, westward IGW, propagating opposite to the mean wind, is also much weaker in the stratosphere in Fig. 5. But after the mean wind reverse above $z \sim 90 \mathrm{~km}$ (see Fig. 3) the westward wave goes upstream, grows, and becomes larger than the eastward IGW. So, one may see in Fig. 5 a peak of eastward wave acceleration near $z \sim 90 \mathrm{~km}$, and a smaller peak of westward wave acceleration at $z \sim 115 \mathrm{~km}$ in summer.

Figure 6 depicts the vertical profiles of the parameters for IGW harmonics with $c= \pm 100 \mathrm{~m} \mathrm{~s}^{-1}$ that greatly exceeds the mean wind in the stratosphere. Such waves do not become saturated and breaking, but may dissipate due to background turbulence, nonlinear destruction of the primary wave into a spectrum of secondary harmonics, and due to other dissipative mechanisms involved in our model (see earlier). Slightly larger dissipation of the westward harmonic in the winter stratosphere can not compensate for the greater effectiveness of the generation of the eastward harmonic by the tropospheric wave source for (see description of Fig. 2), so eastward IGW and the wave acceleration dominate in the top plots of Fig. 6 in the entire middle and upper atmosphere. In summer the mean wind reverse above $z \sim 90 \mathrm{~km}$ makes the westward IGW stronger at high altitudes. Consequently, in Fig. 6 the dominant wave accelerations are eastward below $z \sim 100 \mathrm{~km}$ and westward above $z \sim 100 \mathrm{~km}$ in summer when the tropospheric IGW sources have the same strength in both directions.

\section{Discussion and conclusion}

In this study the parametrization of IGW dynamic and thermal effects developed by Gavrilov (1990) involving the wave generation by mesoscale turbulence in the 

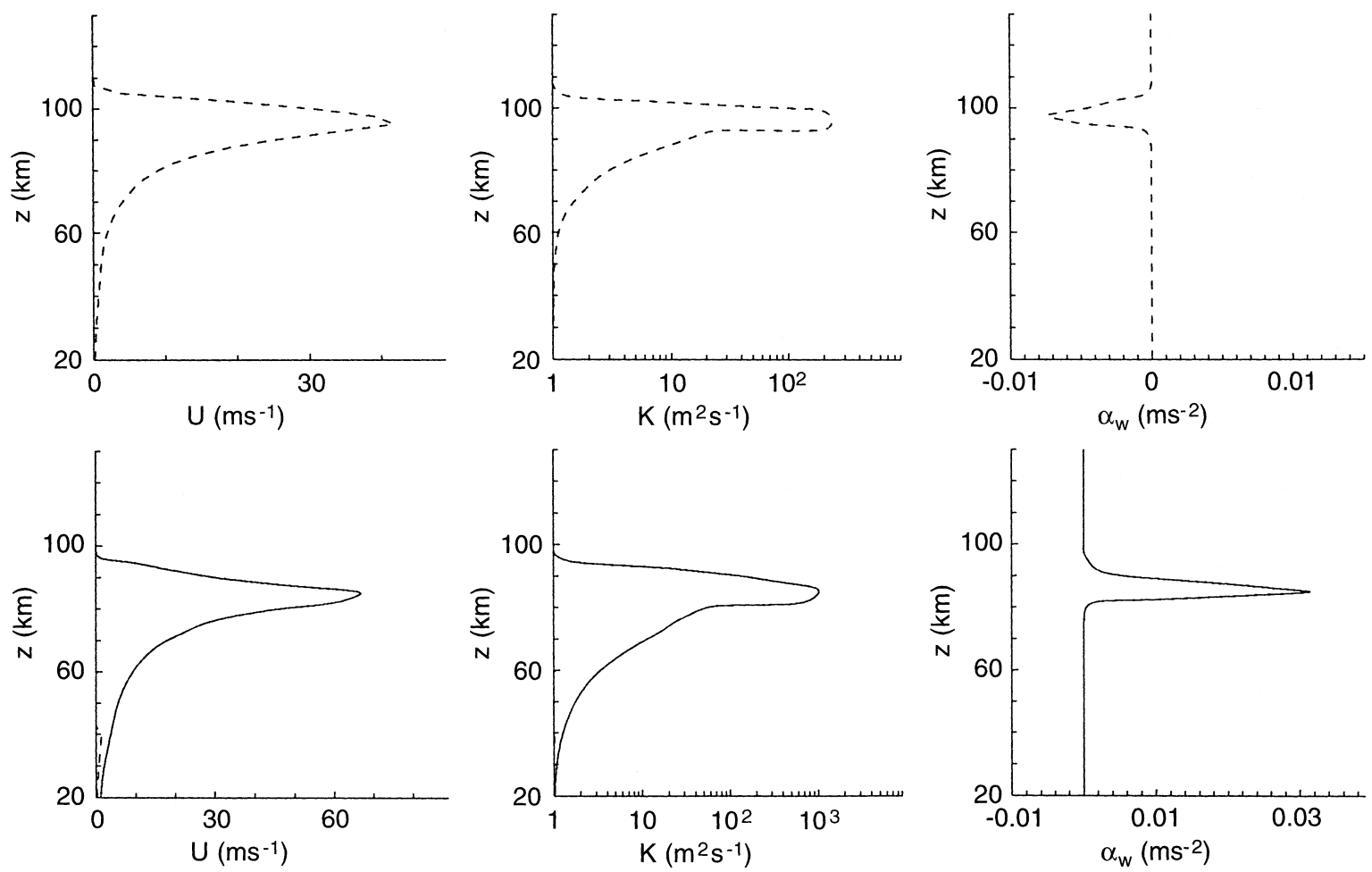

Fig. 4. Vertical profiles of the parameters of IGW harmonics with $|c|=30 \mathrm{~m} \mathrm{~s}^{-1}$ and $\sigma=10^{-3} \mathrm{~s}^{-1}$ propagated eastwards (solid lines), and westwards (dashed lines) in January (top plots) and July (bottom

plots). Tropospheric wave source is the same as in Fig. 1. Definition of the parameters see Fig. 2
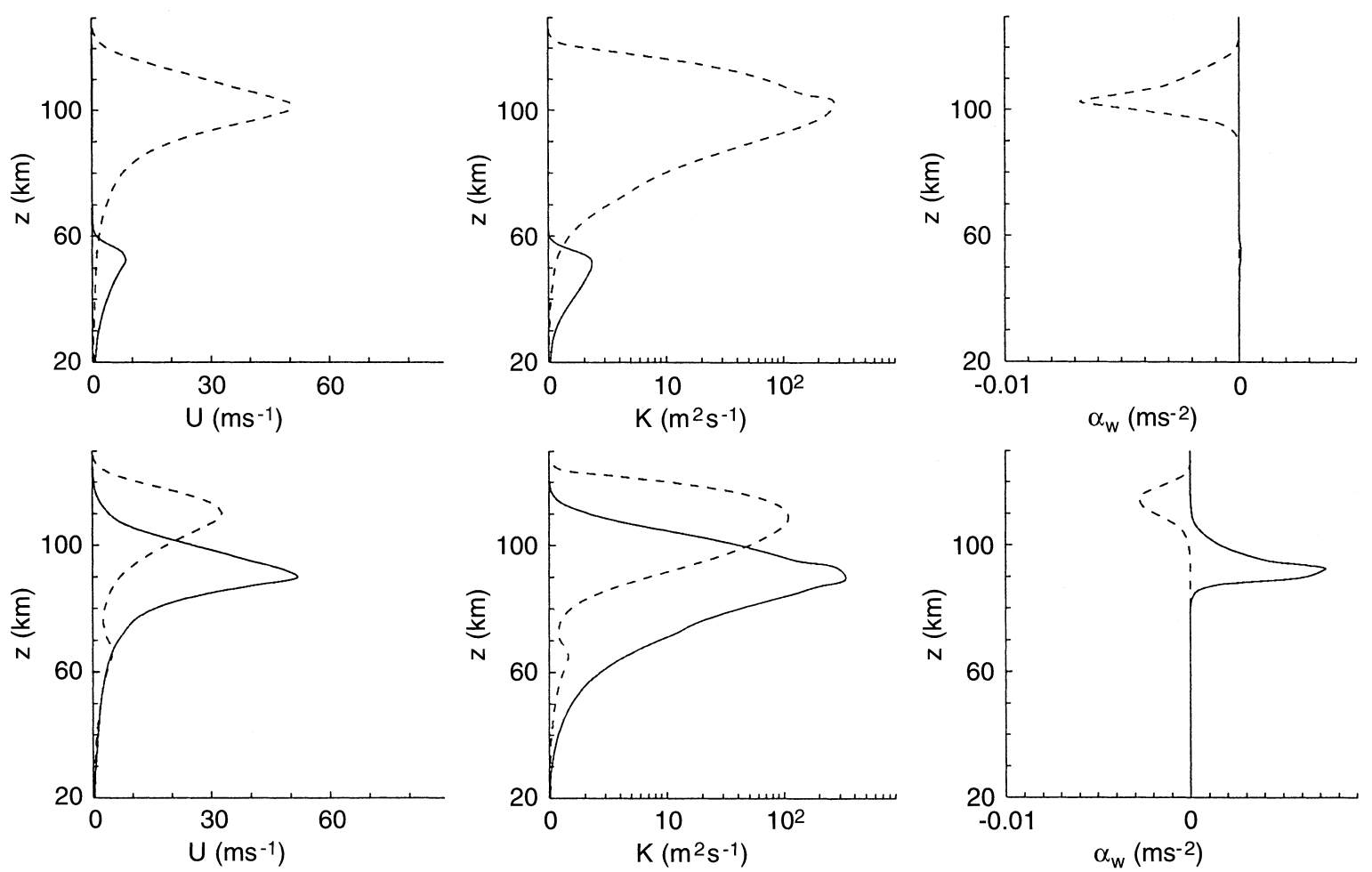

Fig. 5. Same as Fig. 4, but for IGWs with $|c|=60 \mathrm{~m} \mathrm{~s}^{-1}$ 

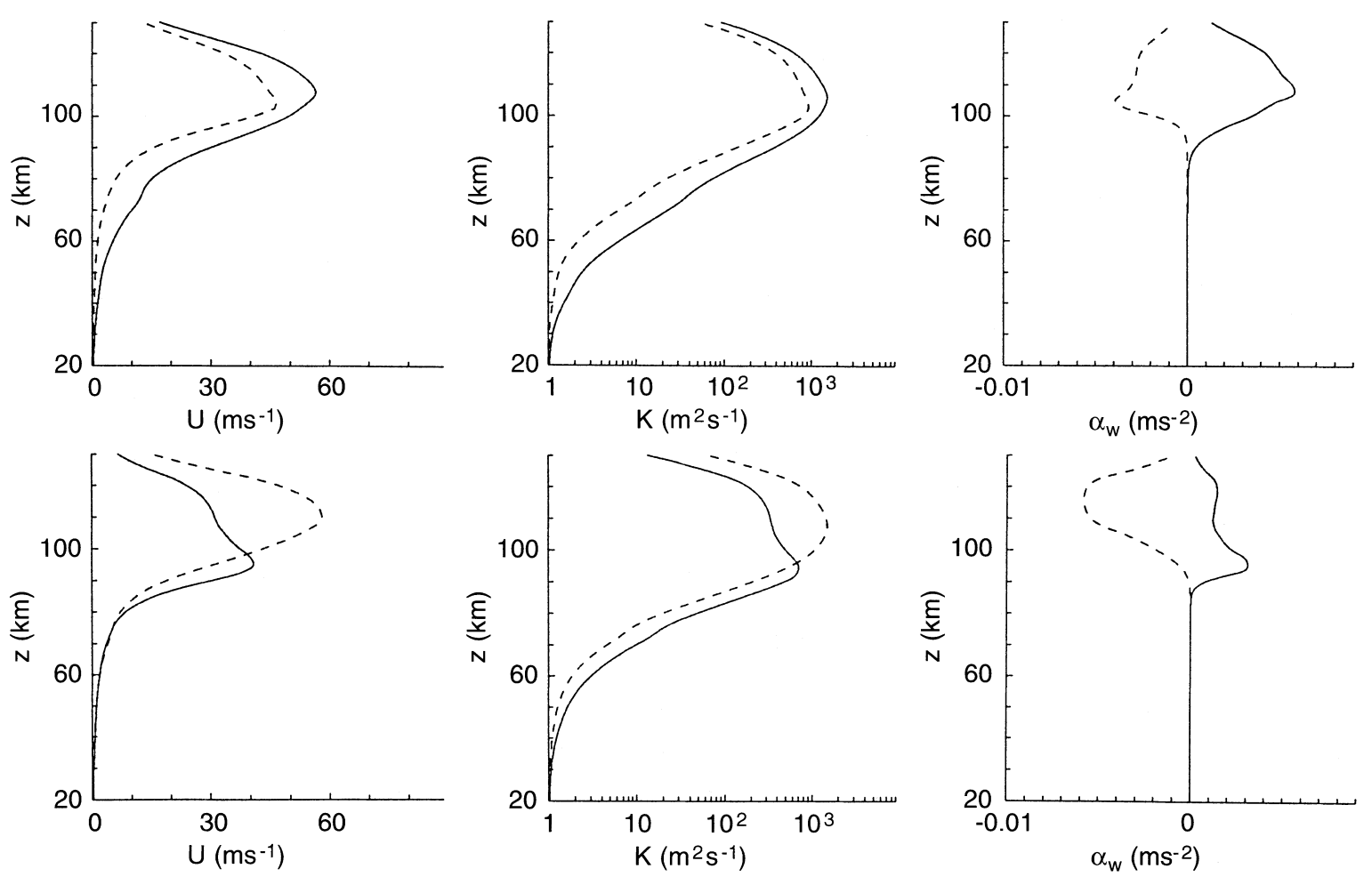

Fig. 6. Same as Fig. 4, but for IGWs with $|c|=100 \mathrm{~m} \mathrm{~s}^{-1}$

troposphere has been improved. The equations that describe the generation of waves by hydrodynamic sources of momentum, heat and mass are derived. To illustrate the properties of developed parametrization, calculations of amplitudes, wave energy fluxes, turbulent viscosities, heating rates, and accelerations of the mean flow caused by IGWs generated in the troposphere have been made. A comparison of different mechanisms of turbulence production in the atmosphere by IGWs shows that the nonlinear destruction of a primary IGW into a spectrum of secondary waves may be essential for nonsaturated stable IGWs.

It is widely supposed now that the wave drag of the mean flow leads to the reverse of zonal wind near $z \sim 100 \mathrm{~km}$ (as in Fig. 4). Our modelling reveals that the resulting direction of the wave acceleration may depend on two mechanisms: the preference of the wave source to generate IGWs propagated in the direction of tropospheric mean wind, and the filtering effects of the mean wind in the middle atmosphere. Stratospheric winds cause stronger dissipation of IGWs propagated opposite to them, and contribute to the formation of westward wave accelerations in winter and eastward accelerations in summer near $z \sim 100 \mathrm{~km}$.

Figure 6 shows that IGWs with large horizontal phase speed are less influenced by stratospheric winds. Usually, the winds in the troposphere are relatively slow, but in some places and at some times they may have substantial speed. Measurements with Japanese MU radar (Murayama et al., 1994) give a mean wind speed up to $60-80 \mathrm{~m} \mathrm{~s}^{-1}$ at altitudes of $10-12 \mathrm{~km}$ in January. Temporarily the wind may have greater speeds. Such strong winds may generate IGWs with large horizontal phase speeds in the troposphere. Also, fast IGWs may be generated inside the stratosphere, which has larger wind speeds. Then these waves may propagate into the upper atmosphere.

The simple numerical scheme developed in this study may be useful for the parametrization of the IGW generation by hydrodynamic wave sources, and of the influence of the waves on the dynamics and thermal regime of the middle atmosphere. The IGW model is made here for a plane monochromatic IGW harmonic, but it may be used for the development of statistical numerical models of IGW spectra. Our calculations are made for arbitrarily chosen values of the strength of tropospheric hydrodynamic wave sources. Further study of the strength of wave sources and its spectral composition is required for the development of more precise parametrizations.

Acknowledgements. This study was supported by the Russian Foundation for Basic Research and International Association for the promotion of cooperation with scientists from the New Independent States (INTAS).

Topical Editor F. Vial thanks L. Pfister and S. Echermann for their help in evaluating this paper.

\section{References}

Bacmeister, J. T., Mountain-wave drag in the stratosphere and mesosphere inferred from observed winds and a simple mountain-wave parametrization scheme, J. Atmos. Sci., 50, 377-399, 1993

Belov, P. N., Numerical methods of weather forecast, Hydrometeoizdat Press, Leningrad, 1975.

Bertin, F., J. Testud, L. Kersley, and P. R. Rees, The meteorological jet stream as a source of medium scale gravity waves in the 
thermosphere: an experimental study, J. Atmos. Terr. Phys., 40, 1161-1183, 1978.

Brodhun, D., G. Bull, and I. Neisser, On the identification of tropospheric sources of gravity waves observed in the mesosphere, Z. Meteorol., 24, 299-308, 1974.

Chunchuzov, I. P., On a possible generation mechanism for nonstationary mountain waves in the atmosphere, J. Atmos. Sci., 51, 2196-2206, 1994.

Dikiy, L. A., Theory of oscillations of the Earth atmosphere, Hydrometeoizdat Press, Leningrad, 1969.

Dong, B., and K. C. Yeh, Resonant and nonresonant wave-wave interactions in an isothermal atmosphere, J. Geophys. Res., 93(D4), 3729-3744, 1988.

Drobyazko, I. N., and V. N. Krasilnikov, Acoustic-gravity wave generation by atmospheric turbulence, Radiophys., Izv VUZ of USSR, 28, 1357-1365, 1985.

Einaudi, F., D. P. Lalas, and G. E. Perona, The role of gravity waves in tropospheric processes, Pure Appl. Geophys., 117, 627663, 1978 .

Fritts, D. C., Shear excitation of atmospheric gravity waves, 2. Nonlinear radiation from a free shear layer, J. Atmos. Sci., 41, 524-537, 1984.

Fritts, D. C., and W. Lu, Spectral estimates of gravity wave energy and momentum fluxes. Part 2: parametrization of wave forcing and variability. J. Atmos. Sci., 50, 3695-3713, 1993.

Fritts, D. C., and Z. Luo, Gravity wave excitation by geostrophic adjustment of the jet stream, Part 1: two-dimensional forcing, $J$. Atmos. Sci., 49, 681-697, 1992.

Fritts, D. C., and G. D. Nastrom, Sources of mesoscale variability of gravity waves, Part 2: frontal, convective and jet stream excitation, J. Atmos. Sci., 49, 111-127, 1992.

Fovell, R., D. Durran, and J. R. Holton, Numerical simulation of convectively generated stratospheric gravity waves, J. Atmos. Sci., 49, 1427-1442, 1992.

Gage, K. S., Evidence for $k^{-5 / 3}$ law inertial range in mesoscale twodimensional turbulence, J. Atmos. Sci., 36, 1950-1953, 1979.

Gavrilov, N. M., On the nonlinear generation of wave motions in the atmosphere by quasi-geostrophic and eddy motions, Meteorol res., Geophys. committee of USSR Acad. Sci. Press, Moscow, 13, 49-71, 1987.

Gavrilov, N. M., On the generation of internal gravity waves in the atmosphere by mesoscale turbulence, Investigations of dynamic processes in the upper atmosphere, Hydrometeoizdat Press, Moscow, 74-77, 1988.

Gavrilov N. M., Parametrization of accelerations and heat flux divergences produced by internal gravity waves in the middle atmosphere, J. Atmos. Terr. Phys., 52, 707-713, 1990.

Gavrilov N. M., Internal gravity waves in the mesopause region: hydrodynamical sources and climatological patterns, $A d v$. Space Res., 12, (10)113-(10)121, 1992.

Gavrilov N. M. and G. M. Shved, On the closure of equation system for the turbulized layer of the upper atmosphere, Ann. Geophysicae, 31, 375-388, 1975.

Gavrilov N. M. and V. A. Yudin, Numerical modelling of the IGW propagation from nonstationary tropospheric sources. Atmos. Oceanic Phys., Izv. USSR Acad. Sci., 23, 241-248, 1987.

Gavrilov N. M. and V. A. Yudin, Model for coefficients of turbulence and effective Prandtl number produced by breaking gravity waves in the upper atmosphere, J. Geophys. Res., 97, 7619-7624, 1992

Gavrilov N. M., S. Fukao, T. Nakamura, M. D. Yamanaka, and M. Yamamoto, On the influence of the mean wind on the anisotropy of internal gravity waves in the middle atmosphere, Ann. Geophysicae, 13, 1355-1356, 1995.

Gavrilov N. M., S. Fukao, T. Nakamura, T. Tsuda, M. D. Yamanaka, and M. Yamamoto, Statistical analysis of gravity waves observed with the MU radar in the middle atmosphere: 1 . Method and general characteristics. J. Geophys. Res., 101(D23), 29511-29521, 1996.

Gill, A., Atmosphere-ocean dynamics, Academic Press, New York, 1982.
Golitsyn, G. S., N. N. Romanova, and E. P. Chunchuzov, On generation of internal waves in the atmosphere by sea-way, Atmos. Oceanic Phys., Izv. USSR Acad. Sci., 12, 319-323, 1976.

Grigoriev, G. I., and O. N. Savina, Transition emission of acousticgravity waves, Radiophy., Izv. VUZ of the USSR, 26, 135-141, 1983.

Grachev, A. I., L. A. Lobachevskiy, L. K. Matveev, M. I. Mordukhovich, O. S. Sergeenko, Thick convective cloudiness as a source of internal waves in the troposphere and ionosphere, Atmos. Oceanic Phys., Izv. USSR Acad. Sci., 20, 173-177, 1984.

Hamilton, K., A study of the occurrence of dynamically unstable conditions in the middle atmosphere, Can. J. Phys., 62, 963967, 1984.

Hines, C. O., A possible source of waves in noctilucent clouds, $J$. Atmos. Sci., 25, 937-942, 1968.

Hines, C. O., Doppler-spread parameterization of gravity-wave momentum deposition in the middle atmosphere. Part 1: basic formulation. J. Atmos. Solar-Terr. Phys., 59, 371-386, 1997.

Holton, J. R., The influence of gravity wave breaking on the general circulation of the middle atmosphere, J. Atmos. Sci., 40, 2497-2507, 1983.

Huang, Y. N., K. Cheng, and S. W. Chen, On the detection of acoustic-gravity waves generated by typhoon by use of real time HF Doppler frequency shift sounding system, Radio Sci., 20, 897-906, 1985.

Jacobson, A. R., and R. C. Carlos, Observations of acoustic-gravity waves in the thermosphere following Space Shuttle ascents, $J$. Atmos. Terr. Phys., 56, 525-528, 1994.

Kazimirovsky, E. S., Effects of thunderstorms in ionospheric processes in Investigations in geomagnetizm, aeronomy and solar phys., Nauka Press, Moscow, 66, 170-192, 1983.

Kersley, L., and P. R. Rees, Tropospheric gravity waves and their possible association with medium-scale travelling disturbances, J. Atmos. Terr. Phys., 44, 147-159, 1982.

Kibel, I. A., On the adaptation of air motion to geostrophic one, Rep. USSR Acad. Sci., 104, 60-63, 1955.

Kulikov, V. V., V. M. Shashunkina, and L. A. Yudovich, Generation of internal gravity waves after the "Skylab" fall, Geomagn. Aeron., 22, 500-502, 1982.

Laprise, J. P. R., An assessment of the WKBJ approximation to the vertical structure of linear mountain waves: implications for gravity-wave drag parameterization, J. Atmos. Sci., 50, 14691487, 1993.

Ley, B. E., and W. R. Peltier, Wave generation and frontal collapse, J. Atmos. Sci., 35, 3-17, 1978.

Lighthill, M. J., On sound generated aerodynamically, 1. General theory, Proc. R. Soc. London, A211, 564-587, 1952.

Lighthill, M. J., Waves in fluids, Cambridge University Press, 1978.

Lilly, D. K., Stratified turbulence and the mesoscale variability of the atmosphere, J. Atmos. Sci., 40, 749-761, 1983.

Lindzen, R. S., Turbulence and stress owing to gravity wave and tidal breakdown, J. Geophys. Res., 86, 9707-9714, 1981.

Lindzen, R. S., Gravity waves in the mesosphere, in Dynamics of the Middle Atmosphere, Dordrecht Press, Tokyo, 3-18, 1984.

Lindzen, R. S., and J. Forbes, Turbulence originated from convectively stable internal waves, J. Geophys. Res., 88(C11), 6549-6553, 1983.

Lipovskiy, V. D., Linear theory of acoustic-gravity waves emission by moving sources, Atmos. Oceanic Phys., Izv USSR Acad. Sci., 17, 1134-1141, 1981.

Lott, F., and H. Teitelbaum, Topographic waves generated by a transient wind, J. Atmos. Sci., 50, 2607-2654, 1993.

Manzini, E., and K. Hamilton, Middle atmospheric travelling waves forced by latent and convective heating, J. Atmos. Sci., 50, 2180-2200, 1993.

Matsuno, T., A quasi-one-dimensional model of the middle atmosphere circulation interacting with internal gravity waves, J. Meterol. Soc. Japan, 60, 215-226, 1982.

McLandress, C., and N. A. McFarlane, Interactions between orographic gravity wave drag and forced stationary planetary 
waves in the winter northern hemisphere middle atmosphere, $J$. Atmos. Sci., 50, 1966-1990, 1993.

Medvedev, A. S., and N. M. Gavrilov, The nonlinear mechanism of gravity wave generation by meteorological motions in the atmosphere, J. Atmos. Terr. Phys., 57, 1221-1231, 1995.

Medvedev, A. S., and G. P. Klaassen, Vertical evolution of gravity wave spectra and the parameterization of associated wave drag, J. Geophys. Res., 100(D12), 25, 841-853, 1995.

Monin, A. S., and A. M. Yaglom, Statistical hydromechanics, Nauka Press, Moscow, 1965.

Murayama, Y., T. Tsuda, and S. Fukao, Seasonal variation of gravity wave activity in the lower atmosphere observed with the MU radar, J. Geophys. Res., 99(D11), 23057-23069, 1994.

Nagpal, O. P., The sources of atmospheric gravity waves, Contemp. Phys., 20, 593-609, 1979.

Nastrom, G. D., and D. C. Fritts, Sources of mesoscale variability of gravity waves, Part 1: topographic excitation, J. Atmos. Sci., 49, 101-110, 1992.

Obukhov, A. M., On the problem of geostrophic wind, Geophys. Geograph., Izv. USSR Acad. Sci., 13, 281-306, 1949.

Pedlosky, J., Geophysical fluid dynamics. Springer-Verlag, New York Berlin Heidelberg, 1982.

Pertsev, N. N., Disturbance of the atmosphere caused by flowing around an isolated mountain, Atmos. Oceanic Phys., Izv. USSR Acad. Sci., 19, 570-575, 1983.

Pfister L., S. Scott, M. Loewenstein, S. Bowen, and M. Legg, Mesoscale disturbances in the tropical stratosphere excited by convection: observations and effects on the stratospheric momentum budget, J. Atmos. Sci., 50, 1058-1075, 1993a.

Pfister, L., K. R. Chan, T. P. Bui, S. Bowen, M. Legg, B. Gary, K. Kelly, M. Proffitt, and W. Starr, Gravity waves generated by a tropical cyclone during the STEP tropical field program: a case study, J. Geophys. Res., 98, 8611-8638, 1993b.

Roberts, D. H., J. A. Klobuchar, P. F. Fougere, and D. H. Hendricson, Large-amplitude travelling ionospheric disturbance produced by the May 18, 1980 explosion of Mount St. Helene, J. Geophys. Res., 87, 6291-6301, 1982.

Rossby, C. G., On the mutual adjustment of pressure and velocity distribution in certain simple current systems, J. Mar. Res., 1, 15-28, 1937.
Rozenfeld, S. H., On the damping of internal gravity waves in the atmosphere due to generation of secondary harmonics, Atmos. Oceanic Phys., Izv. USSR Acad. Sci., 19, 1011-1019, 1983.

Samardjiev, D., and C. Pashova, Wave ionospheric disturbances observed after earthquake on 4.III.1977 at Vrancha, Bulg. Geophys. Descript., 8, 44-51, 1982.

Schaning B., Long-term variation of thunderstorm activity over the Amazon basin during southern summer as derived from atmospheric noise measurements. J. Atmos. Terr. Phys., 54, 1329-1336, 1992.

Schoeberl, M. R., The penetration of mountain waves into the middle atmosphere, J. Atmos. Sci., 42, 2856-2864, 1985.

Somsikov, V. M., On mechanisms for the formation of atmospheric irregularities in the solar terminator region, J. Atmos. Terr. Phys., 57, 75-84, 1995.

Sonmor, L. J., and G. P. Klaassen, Higher-order resonant instabilities of internal gravity waves, J. Fluid Mech., 324, 123, 1996.

Stein, R. S., Generation of acoustic and gravity waves by turbulence in an isothermal stratified atmosphere, Solar Phys., 2, 285-432, 1967.

Sutherland, B. R., C. P. Caulfield, and W. R. Peltier, Internal gravity wave generation and hydrodynamic instability, $J$. Atmos. Sci., 51, 3261-3280, 1994.

Townsend, A. A, Excitation of internal waves by a turbulent boyndary layer, J. Fluid. Mech., 22, 241-252, 1965.

Townsend, A. A., Internal waves produced by a convective layer, $J$. Fluid. Mech., 24, 307-319, 1966.

Varshavskiy, I. I., and A. D. Kalikhman, Ionospheric effects of ground industrial explosions, Geomagn. Aeron., 24, 211-216, 1984.

Weinstock, J., Nonlinear theory of gravity waves: momentum deposition, generalized Rayleigh friction, and diffusion, $J$. Atmos. Sci., 39, 1698-1710, 1982.

Weinstock, J., Saturated and unsaturated spectra of gravity waves and scale-dependent diffusion, J. Atmos. Sci., 47, 2211-2225, 1990. 